\title{
REVIEW
}

\section{Deficits in attention, motor control, and perception: a brief review}

\section{Gillberg}

The concept of DAMP (deficits in attention, motor control, and perception) has been in clinical use in Scandinavia for about 20 years. DAMP is diagnosed on the basis of concomitant attention deficit/hyperactivity disorder and developmental coordination disorder in children who do not have severe learning disability or cerebral palsy. In clinically severe form it affects about $1.5 \%$ of the general population of school age children; another few per cent are affected by more moderate variants. Boys are overrepresented; girls are currently probably underdiagnosed. There are many comorbid problems/overlapping conditions, including conduct disorder, depression/anxiety, and academic failure. There is a strong link with autism spectrum disorders in severe DAMP. Familial factors and pre- and perinatal risk factors account for much of the variance. Psychosocial risk factors appear to increase the risk of marked psychiatric abnormality in DAMP. Outcome in early adult age was psychosocially poor in one study in almost $60 \%$ of unmedicated cases. There are effective interventions available for many of the problems encountered in DAMP.

Correspondence to: Prof. C Gillberg, Department of Child and Adolescent Psychiatry, Göteborg Unviersity, Kungsgatan 12, 41119 Göteborg, Sweden; christopher.gillberg@ pediat.gu.se

Accepted

18 February 2003

......
A ttention deficits, inappropriate overactivity, and problematic impulsiveness are common symptoms causing concern among school age children. ${ }^{1}$ The constellation of problems comprised under the DSM-IV diagnostic category of attention deficit/hyperactivity disorder (ADHD) is the most frequent condition encountered in child psychiatric clinic attenders. ${ }^{2}$ ADHD is the most prevalent among all the child neuropsychiatric/neurodevelopmental disorders. It has been shown to occur at a relatively stable rate of $3-6 \%$ of all young children in Sweden, Denmark, the USA, Australia, Spain, and Brazil, to mention only a few of the countries in which epidemiological studies have been performed. ${ }^{3}$

ADHD is now a widely accepted diagnostic concept that has replaced the rather less precise category of minimal brain dysfunction (MBD), a term almost universally employed in child psychiatry and developmental paediatrics from the 1950s to the early 1980s. In its DSM-III-R and DSM-IV versions, ADHD has been the subject of many thousand empirical studies. ADHD overlaps with other disorders, conditions, and problems at an extremely high rate. This overlap is usuallysometimes erroneously-referred to as "comor- bidity". In the past, the MBD label allowed for the comorbidity under the umbrella catch-all etiquette. The gradual takeover of ADHD as the accepted terminology in the field has led to a purported "purification" of the scientific studies in the field, such that, unfortunately, the overlap with other problems has been ignored. In particular, students of ADHD have neglected the very substantial overlap with motor control dysfuncition. The frequent co-occurrence of psychiatric problems in ADHD has slowly become acknowledged, but the high rate and clinical significance of coexisting developmental incoordination problems (and, to some extent, other specific developmental problems, such as speechlanguage dysfunction) have been virtually ignored.

In Scandinavia, the DAMP (deficits in attention, motor control, perception) concept was developed in the 1970s, in an attempt to operationalise the syndrome of $\mathrm{MBD}$, long before the publication of the DSM-III-R (in which ADHD appeared in a formalised fashion for the first time). About 30 years ago, MBD in Scandinavia, just as in the rest of the world, was conceptualised as a combination of several dysfunctions in the field of attention, activity regulation, impulse control, motor control, learning problems, speech and language difficulties, and perceptual abnormalities. ${ }^{4}$ Bengt Hagberg, world famous expert in child neurology, suggested the embryo of an operationalised variant of the disorder. ${ }^{5}$ In 1974 he initiated a large scale empirical study of MBD. This initiative led to several other studies out of which gradually grew the concept of DAMP. While the MBD term was gradually discarded (after influential critical papers), ${ }^{67}$ the DAMP concept became rooted in everyday clinical practice and parlance, particularly in Denmark and Sweden. ${ }^{89}$

This review draws on all the published studies (original contributions and book chapters) referring to the DAMP syndrome (about 50 in total). The vast majority of these have either originated in the author's own clinical and research setting or have been supervised and/or co-authored by him. A small number of studies and book chapters have come from the USA, the UK, Australia, and Denmark. Inevitably, some of the conclusions and clinical recommendations lean

Abbreviations: ADHD, attention deficit/hyperactivity disorder; DAMP, deficits in attention, motor control, and perception; $D C D$, developmental coordination disorder; $H K D$, hyperkinetic disorder; $M B D$, minimal brain dysfunction; ODD, oppositional defiant disorder 


\section{Box 1 Diagnostic criteria for DAMP}

(A) ADHD as defined in the DSM-IV

(B) DCD as defined in the DSM-IV

(C) Condition not better accounted for by cerebral palsy

(D) Not associated with severe learning disability-that is, IQ should be higher than about 50

(E) Other diagnostic categories often apply (for example, autism spectrum disorder, ODD, depression), but are not required for diagnosis of DAMP

on the vast literature on ADHD, and on the 30 years of clinical experience on the part of the author in dealing with problem children with DAMP and their families. It could be argued that with several thousand papers published on ADHD, the database for conclusions in the field of DAMP is weak, relatively speaking. However, this would be equally true of the much discussed concept of ICD-10 hyperkinetic disorder (HKD), which has a very limited number of publications to its name.

The DAMP acronym may have negative connotations in English speaking countries, particularly if used in connection with children. There is also the very real issue of how to deal with the conflict between splitting (ADHD plus developmental coordination disorder (DCD)) and lumping (DAMP). Nevertheless, the DAMP construct has been helpful in identifying a group of children with ADHD and multiple needs that will not be self evident if the diagnosis is just ADHD or just DCD. There appears to be an interactive effect between the two, and children with the combination have much more academic problems and autistic type behaviours than would be suggested by having the added effects of the two separate conditions

\section{DEFINITIONS}

In the first longitudinal population based study of DAMP (originally referred to as "MBD" and "perceptual, motor, and attentional deficits"), the condition was defined as the combination of: (1) cross situational impairing attention deficit (ADD), with or without impairing hyperactivity/impulsivity; and (2) impairing deficit in at least one of the following areas: gross motor, fine motor, perception, or speech-language, in the absence of clear mental retardation and cerebral palsy/other major neurological disability. Severe DAMP was diagnosed in cases showing the combination of (1) and all of the deficits listed under $(2) .^{10}$

In later reports from other population based surveys, after the original study had shown that children with perceptual abnormality virtually always had some impairing motor control problems, and in order to comply with emerging international diagnostic consensus practice, DAMP has been defined as the combination of ADHD and DCD (developmental coordination disorder) ${ }^{11}$ (boxes $1-3$ ). Defined in this way, DAMP constitutes a subgroup of the diagnostic category of ADHD, conceptually similar-but not clinically identical-to the WHO concept of HKD (hyperkinetic disorder).

\section{PREVALENCE}

DAMP in severe form occurs in $1.2-2.0 \%$ of all 7 year olds. ${ }^{10}{ }^{11}$ An additional $3-6 \%$ of the general population in that age group have milder variants of the disorder. ${ }^{12}$ According to Kadesjö and Gillberg, ${ }^{13}$ DAMP constitutes about half of all cases of ADHD. The severe form of DAMP is always clinically impairing and has usually led to attendance at paediatric, child psychiatric, child neurological, or speech language clinics before age 10 years (often with a referral diagnosis of DAMP, ADHD, HKD, "conduct disorder", or "autism spectrum disorder"). In the milder variants there is also commonly a requirement for specialist evaluation/intervention, but quite
Box 2 Diagnostic criteria for attention deficit/hyperactivity disorder*

(A) Either (1) or (2)

(1) Six (or more) of the following symptoms of inattention have persisted for at least six months to a degree that is maladaptive and inconsistent with developmental level:

Inattention

- (a) Often fails to give close attention to details or makes careless mistakes in schoolwork, work, or other activities

- (b) Often has difficulty sustaining attention in tasks or play activities

- (c) Often does not seem to listen when spoken to directly

- (d) Often does not follow through on instructions and fails to finish schoolwork, chores, or duties in the workplace (not due to oppositional behaviour or failure to understand instructions)

- (e) Often has difficulty organising tasks and activities

- (f) Often avoids, dislikes, or is reluctant to engage in tasks that require sustained mental effort (such as schoolwork or homework)

- (g) Often loses things necessary for tasks or activities (for example, toys, school assignments, pencils, books, or tools

- (h) Is often easily distracted by extraneous stimuli

- (i) Is often forgetful in daily activities

(2) Six (or more) of the following symptoms of hyperactivity impulsivity have persisted for at least six months to a degree that is maladaptive and inconsistent with developmental level:

Hyperactivity

- (a) Often fidgets with hands or feet or squirms in seat

- (b) Often leaves seat in classroom or in other situations in which remaining seated is expected

- (c) Often runs about or climbs excessively in situations in which it is inappropriate (in adolescents or adults may be limited to subjective feelings of restlessness)

- (d) Often has difficulty playing or engaging in leisure activities quietly

- (e) Is often "on the go", or often acts as if "driven by a motor"

- (f) Often talks excessively

Impulsivity

- (g) Often blurts out answers before questions have been completed

- (h) Often has difficulty awaiting turn

- (i) Often interrupts or intrudes on others (for example, butts into others' conversations or games)

(B) Some hyperactive-impulsive or inattentive symptoms that caused impairment were present before age 7 years (C) Some impairment from the symptoms is present in two or more settings (for example, at school (or work) and at home)

(D) There must be clear evidence of clinically significant impairment in social, academic, or occupational functioning

(E) The symptoms do not occur exclusively during the course of a pervasive developmental disorder, schizophrenia, or other psychotic disorder, and are not better accounted for by another mental disorder (for example, mood disorder, anxiety disorder, dissociative disorder, or a personality disorder)

Code based on type

- 314.01-Attention deficit/hyperactivity disorder, combined type: if both criteria $\mathrm{A} 1$ and $\mathrm{A} 2$ are met for the past six months

- 314.00-Attention deficit/hyperactivity disorder, predominantly inattentive type: if criterion $A 1$ is met but criterion $A 2$ is not met for the past six months

- 314.01-Attention deficit/hyperactivity disorder, predominantly hyperactive-impulsive type: if criterion A2 is met but criterion $A 1$ is not met for the past six months

Coding note

For individuals (especially adolescents and adults) who currently have symptoms that no longer meet full criteria, "in partial remission" should be specified.

*American Psychiatric Association (1994). 
Box 3 Diagnostic criteria for developmental coordination disorder*

(A) Performance in daily activities that require motor coordination is substantially below that expected given the person's chronological age and measured intelligence. This may be manifested by marked delays in achieving motor milestones (for example, walking, crawling, sitting), dropping things, "clumsiness," poor performance in sports, or poor handwriting

(B) The disturbance in criterion A significantly interferes with academic achievement or activities of daily living

(C) The disturbance is not due to a general medical condition (for example, cerebral palsy, hemiplegia, or muscular dystrophy) and does not meet criteria for a pervasive developmental disorder

(D) If mental retardation is present, the motor difficulties are in excess of those usually associated with it.

\section{Coding note}

If a general medical (for example, neurological) condition or sensory deficit is present, code the condition on axis III.

*American Psychiatric Association (1994).

often at slightly later ages, and almost invariably in a plethora of diagnostic disguises (including "depression", "anxiety disorder", "conduct disorder", or "academic failure"). ${ }^{14}$

\section{MALE:FEMALE RATIOS}

Most of the studies of DAMP have reported a male:female ratio of 3-5:1, which is similar to ratios found in other neuropsychiatric/neurodevelopmental disorders. It is possible that girls with DAMP (and with ADHD and DCD) are often missed or misdiagnosed (for example, as "depression" or "anxiety disorder"). ${ }^{14}$

\section{OVERLAP OF ADHD AND DCD}

As has already been mentioned, about half of all cases with ADHD meet criteria for DAMP - that is, they have DCD. ${ }^{13}$ Conversely, ADHD occurs in about half of all cases of DCD. It is interesting to note that DCD in severe or moderate form occurs at similarly high rates in both severe and moderate forms of ADHD. According to one study, ${ }^{15}$ this is very different from the overlap pattern of ADHD with oppositional defiant disorder (ODD): about $60 \%$ of cases with severe ADHD meet criteria for ODD, but only about $10 \%$ of moderate ADHD cases show this type of comorbidity. This might signal a more robust overlap of ADHD with DCD, and provide an indication that ODD may be a measure of severity in ADHD rather than a comorbid syndrome. Interestingly, a weaker pattern of overlap is seen with the $\mathrm{AD}$ and HD subgroups of ADHD: less than half of all cases meeting criteria for $\mathrm{AD}$ (the inattentive group) also meet criteria for HD (the hyperactive-impulsive group).

\section{OTHER COMORBIDITIES/OVERLAPPING PROBLEMS}

Data on comorbidity in DAMP stem from the four epidemiological studies, all of which are Swedish and span the time period from the mid 1970s to the mid 1990s. In the first study (from Gothenburg in the 1970s), DAMP was shown to overlap with a number of other diagnostic entities. ${ }^{16}$ Already at age 7 years, about one in three children with the combination of ADHD and DCD (DAMP) met criteria for depressive disorder, and one in ten had shown enough antisocial behaviour to qualify for a diagnosis of conduct disorder. These two types of problems occurred in children with severe and mild to moderate variants alike. In those with severe DAMP, autistic features were extremely common, amounting to what would nowadays be diagnostic status for "autism spectrum disorder" in no less than two thirds of cases. Several of the cases in this subgroup-constituting about $0.7 \%$ of the general population of Swedish 7 year olds in the mid-1970s-met full operationalised criteria for Asperger's syndrome, as shown in a separate study performed in the 1980s. ${ }^{17}$ The later population study from Karlstad showed convincingly that DAMP, but not so much ADHD or DCD separately, tended to predict a high risk of autistic type problems. It is interesting to note that autism spectrum disorders were as common in Sweden in the 1970s as is reported today from the UK and USA, where many discuss the possibility of a recent rise in autism prevalence. This flies in the face of reports from the UK, and, particularly the USA, of a veritable "autism epidemic". Altogether $65 \%$ of individuals with DAMP (severe and mild to moderate variants included) had some kind of "marked psychiatric abnormality" at age 7 years.

Learning problems, including reading-writing disorder and mathematics disorder, were present in $65-80 \%$ of DAMP cases both at age 10 years, and later at age 13 years. Speech and language disorders (ranging from receptive-expressive disorder and semantic-pragmatic disorder to dysarticulation and stuttering) were present in half of all individuals with DAMP. In accordance with the definition of severe DAMP (see above), all those with this variant of the condition had speech-language disorder, whereas in those with mild to moderate variants, about one in four had such a disorder.

Two in three with DAMP met criteria for DSM-III-R psychiatric disorder (other than ADHD and DCD) or personality disorder at age 16 years. A similar proportion met such criteria at age 22 years also. Among the diagnoses that were most prevalent at ages 16 and 22 years were antisocial personality disorder, substance use disorder, and bipolar disorder.

Tic disorders (including Tourette syndrome) are also commonly encountered in ADHD, with and without DCD. ${ }^{15}$ It appears that the link here is with ADHD rather than with DCD (with or without ADHD)

\section{THE COURSE OF DAMP}

Children diagnosed at early school age as having DAMP have a very high risk of persistent problems of various kinds throughout childhood and adolescence, and well into adulthood. Almost $60 \%$ of those with a diagnosis of DAMP at age 7 years had a "very poor outcome" at age 22 years in the Gothenburg longitudinal study. ${ }^{18}$ Thus, they were either receiving a full sick pension/had been on sick leave for a year or more, suffered from severe persistent personality or psychiatric disorder (depression not included), had substance abuse, or had committed serious criminal offences at the time of examination. The proportion of very poor outcome among those with DAMP was more than four times that of the general population without DAMP. In addition, the DAMP group with very poor outcome on average had two or more indications of poor outcome, whereas those without DAMPwhen outcome was poor-usually only had one such indication.

Motor clumsiness tends to become much less markedcertainly much less obvious-with increasing age. Already at age 10 years, obvious motor clumsiness (which was present in all cases around age 7 years) is no longer easily discovered in more than about half of all cases. This proportion drops to about one in three by the early teens. DCD can then be diagnosed in $30-35 \%$ of the original DAMP cases (and not at all in non-DAMP, non-DCD cases) at the ages of 16 and 22 years. Thus, there is an impression of improved motor skill control in the DAMP group over time. However, there are indications that it is the crudeness of the diagnostic evaluation that leads to the conclusion of better motor skills with age rather than any real improvement relative to a meticulously examined control group. ${ }^{19}$ Nevertheless, DCD in itself does not appear to have the same clinical impact on adult people that it does at younger ages..$^{20}$ 
Attention deficits tend to persist into adult age in a majority of all DAMP cases. ${ }^{18}$ However, only about one in two meet full criteria for ADHD around age 22 years. This may be because overt symptoms of overactivity and impulsivity tend to decline in frequency and impact over the years, even in those cases that show persistent impairing attention deficits well into the 20 s.

Attempts to tease out the contribution of background factors to the very poor psychosocial early adult outcome in untreated cases of DAMP have not been very successful. It appears that DAMP in itself-if untreated-predisposes to psychiatric disorders, antisocial development, and substance abuse in early adult life. It may merit mention here that none of the cases in the longitudinal prospective controlled follow up study of DAMP in Gothenburg, Sweden, had ever received treatment with central stimulants. Many (about 60\%) had consulted specialists (most commonly child psychiatrists) at one or other point during childhood or adolescence, but fewer than a handful had received any kind of intervention, taking the basic dysfunctions (attention deficits, motor control problems, perceptual dysfunction) into account.

Preliminary data from an ongoing follow up study indicate that DCD in boys may constitute a much more handicapping problem as regards psychosocial wellbeing than currently appreciated. Boys with motor control problems have particularly poor self-esteem (Landgren et al, in progress). DCD in ADHD may signal a considerably more severe variant of the disorder, ${ }^{13}$ with stronger links to autism spectrum disorders and academic failure than in ADHD cases without concomitant DCD. The link with poor self-esteem may be an important predictor of poor outcome, but, at the present time, this conclusion represents conjecture rather than being systematically empirically evidence based.

The natural outcome in the longer term perspective (into the 30s and middle age) of DAMP is not known. However, retrospective studies have shown that DAMP exists and can be reliably diagnosed, even beyond age 50 years (Råstam et al, in progress).

\section{RISK FACTORS FOR THE DEVELOPMENT OF DAMP}

Familial factors play an important role in DAMP. About one in two of all children with the condition have a sibling or parent similarly affected, even though the degree of severity of symptoms varies considerably across family members. A brother may have ADHD combined subtype, a mother may have ADHD mainly inattentive subtype, and a father may have DCD. According to two different studies, familial factors were the main possible aetiological links in about one third of the cases. $^{21} 22$

Various pre- and perinatal risk factors are much overrepresented in DAMP. Low birth weight and premature birth have repeatedly been shown to be associated with $\mathrm{ADHD}_{1}^{23}$ and have also been implicated in DAMP. ${ }^{21}$ Maternal alcohol abuse in pregnancy appears to be associated with a much increased risk of DAMP in the offspring..$^{24}$ Equally, smoking in pregnancy probably has a separate effect on the odds for DAMP or ADHD developing in the child. ${ }^{22}{ }^{25}$ In one of the Swedish studies, ${ }^{22}$ the risk of speech-language disorder was even higher than the risk of DAMP in the offspring of cigarette smoking mothers.

The combination of familial and pre- and perinatal factors is also common in the background of DAMP cases. There is often a parent with a "shadow" ADHD syndrome and a preand perinatal history of risk factors known to increase the liability of DAMP.

Psychosocial risk factors are also much overrepresented in DAMP. However, in multiple regression analyses, they do not appear to account for the condition as such, but rather for many of the comorbid psychiatric, behavioural, and emotional problems. ${ }^{1621}$ Thus, for instance, a cluster of "non-optimal rearing conditions" (including the use of highly inconsistent patterns of rearing on the part of the parents) did not contribute to the variance of DAMP, but strongly predicted "marked psychiatric abnormality" in both DAMP and non-DAMP cases.

\section{HYPOTHESES ABOUT THE AETIOLOGY OF DAMP}

ADHD is sometimes associated with dopamine dysfunction. ${ }^{26}$ The positive clinical effects of certain central stimulants known to affect the function of the dopamine system have long been taken to support the notion of such dysfunction in ADHD. Recently, several genetic studies have shown the dopamine transporter protein, dopamine D2 receptor, and dopamine D4 receptor genes to be dysfunctional in ADHD. ${ }^{26}$ Nevertheless, these dysfunctional dopamine genes probably account for only a small fraction of the variance of ADHD in the general population. New studies have provided evidence for a link between ADHD and abnormalities on chromosome $16 p .{ }^{28}$ It is possible that the basic neurobiology of ADHD involves several other brain circuitries using transmitters and neuromodulators other than dopamine (noradrenaline, glutamate, etc), but the evidence in this field is only slowly emerging. Motor control problems are likely to be linked to dopamine dysfunction. However, overall, there is very little evidence in the field of DAMP when it comes to basic neurobiology, even though preliminary studies of hand motor functions have provided interesting clues. ${ }^{29}$

\section{NEUROPSYCHOLOGY OF DAMP}

Even though DAMP was originally diagnosed only in cases without learning disability, it has now become clinically accepted to diagnose the condition in mildly mentally retarded individuals. One of the population studies of DAMP (or of ADHD and DCD) showed that a non-verbal IQ under 70 occurred in about one in six cases of ADHD with DCD. ${ }^{15}$ This is similar to the proportion of individuals with DAMP, who-in spite of not testing in the retarded non-verbal range on school entry-attend classrooms for pupils with learning disability for a longer or shorter period during the first nine school years.

Children with ADHD (including the subgroup with DAMP) have an average IQ 5-8 points lower than that of the general population. ${ }^{3}$ The neuropsychology of DAMP is similar to that encountered in ADHD, even though tests of visual-motor perception may show higher rates of dysfunction. ${ }^{30}$ Executive dysfunction is almost universal-just as in other neurodevelopmental/neuropsychiatric disorders. It appears that one subgroup with DAMP (about half of all cases) have the typical troughs on two or more of the four WISC subtests (arithmetic, digit span, digit symbol, and information), whereas another subgroup are depressed on most, if not all subscores. It has been argued that the syndromal condition of DAMP could occur either as the result of a "real" ADHD (with DCD) syndrome or as the consequence of "misunderstood" subnormal intelligence. It is likely that in society today, subnormal intelligence is the most unacceptable of all "diagnoses" and that ADHD/DAMP may be more "interesting sounding". Certainly, a child with subnormal intelligence, submitted to all the demands that mainstream schools make on children, will show "attention deficits", "motor clumsiness", and "perceptual problems". The clinical presentation may be inseparable from that of a child with specific executive function deficits affecting results on the WISC subtests mentioned.

\section{DIAGNOSTIC ASSESSMENTS}

All children with severe DAMP will need a diagnostic appraisal at some point during development, often by a multidisciplinary team of developmental pediatricians/child psychiatrists, clinical psychologists, speech-language therapists, physiotherapists, and occupational therapists, as well as by a special 


\section{Box 4 Diagnostic assessment and evaluation in DAMP}

\section{All cases}

- Detailed developmental history from primary carer

- ADHD-R interview ${ }^{3}$

- Comorbidity questionnaire, for example, "Five to Fifteen" (Kadesjö et al, in progress) or "Child Behavior Checklist"32

- Brief neuromotor examination, for example, that described by Gillberg et al, ${ }^{33}$ or "Medical Motor Examination"13

- Brief general physical examination including height, weight, head circumference, vision and hearing, and screen for multiple minor physical anomalies, for example, that described by Waldrop and Halverson ${ }^{34}$

\section{Severe cases}

As above, plus as appropriate:

- EEG (in cases raising any suspicion of absences)

- Karyotype (for example, if multiple minor physical anomalies, very tall stature to rule out sex chromosome aneuploidies)

- Targeted DNA analysis (for example, to rule out 22q11 deletion syndrome)

- Brain imaging in some cases (for example, when there is doubt as to whether problems started in a fairly abrupt fashion or follow a deteriorating course, suggesting non-developmental underlying cause)

education teacher. Many of those with moderate and milder variants of the condition will also need evaluation, but many of these may be appropriately dealt with in primary services-for example, by school health officers, interested general practitioners, or paediatricians.

The basic evaluation in all cases raising suspicion of suffering from ADHD, DCD, or the combination of the two (= DAMP) should consist of a thorough developmental history (usually from one of the parents), focusing on symptoms of inattention, hyperactivity, impulsivity, autism spectrum problems, tics, conduct problems, motor control dysfunction, speech and language problems, academic failure, and depression. An individual examination of the child, including a brief neurodevelopmental battery, is required in all cases. The motor examination should focus on functional aspects, such as hopping on one leg, standing on one leg, walking on lateral aspects of feet, diadochokinesis, and cutting out a predrawn circle from a piece of paper. The perceptual problems may be disclosed on specific perceptual tests (such as the Southern California Sensory Integration Tests) or on visuoperceptual subtests of the WISC and various simple design copying tasks. Box 4 gives a full outline of assessment and evaluation procedures in DAMP.

\section{INTERVENTIONS}

Except in respect of psychopharmacology, the evidence basis for intervention in ADHD/DAMP is scant. ${ }^{35}$ Nevertheless, based on 30 years of experience in the field, it seems clear to the present author that school age children with severe DAMP are in need of comprehensive evaluation, intervention, and sometimes a long term treatment programme provided by a multidisciplinary team of experts. Many with moderate, and some of those with mild problems, will need similar interventions. Judging from clinical experience, quite a number of those with ADHD without DCD, will not need interventions, and may have a fair or good natural outcome. The fact that so few of those with DAMP do well in the long term without specific help, is one of the main reasons why the concept of DAMP may be helpful in clinical practice.

\section{Psychoeducation}

All families with a child afflicted by DAMP will need psychoeducational intervention of one kind or another. Parents need information about the condition, both with the child present and separately (so that they might bring to the surface sensitive information that they may not want to share with the child). Parents and experts often ask when is the right time to inform the child about his/her condition. The answer is almost always that as soon as a definite diagnosis has been made, the child is the one person "most entitled" to the diagnosis. Just as in matters of adoption and suchlike when we now argue that it might not be a good thing to wait for the child to mature before providing the salient information, so should we be arguing in the field of neurodevelopmental disability. Everything that has a name is less frightening than the unmentionable. It is not that young children with DAMP are not aware that something is amiss. On the contrary, they have been told, time and again, by peers, siblings, parents, and other relatives that their attention deficits, clumsiness, and learning problems are, indeed, major problems, only they (and their nearest and dearest) do not have an umbrella term for them. Better then to have a diagnosis, "a label" to account for the difficulties. It is important to convey the message that neither the child, nor anyone else can be blamed for the condition. Taking away the stigma of DAMP by informing everyone involved (including siblings and important teachers, sometimes others also) about the neurodevelopmental nature of the condition is perhaps the one most important intervention a doctor or psychologist can make. "Upgrading" the family to a level of current expert opinion of DAMP, ADHD, DCD, and comorbidities, is essential in all cases. Lay people often ask: "Why make the diagnosis if you are not intending to provide a treatment?" The simple answer is: "Psychoeducation is the first and most important part of any intervention plan!"

\section{Special educational needs}

Most children with DAMP will need some adjustment in their school setting. This can often be accomplished merely by informing the teacher about the nature of the child's problems. Attending a special classroom may be indicated for parts or all of the school day. A high ratio of teacher to child is almost always a necessary requirement if the child with DAMP is going to progress academically. Keeping on task is almost impossible without an interested, well informed teacher around to get the child "back on track". Most children with DAMP will manage school work much better if they are allowed more time on the tasks allocated, but they also need to have regular brief breaks in order to be able to concentrate at all. Having a "coach" to guide the child through everyday activities-particularly in the school setting-is often one of the best ways to address the many-faceted problems faced by children suffering from DAMP.

\section{Motor control interventions}

About half of all individuals with DAMP need special programmes for the treatment/alleviation of motor control problems. As with other teachers, the most important part of the intervention is to inform the physical education teacher about the child's motor control (and other) problems. Physical education in a small group of "like minded" children may often solve the major issues of not wanting to participate at all, and of making up all sorts of excuses that may be more or less transparent. Children with DAMP comprise the largest subgroup of all those who abstain/refuse/do not participate in physical education. ${ }^{36}$ A physiotherapist or an occupational therapist may need to be involved to make a detailed evaluation of gross or fine motor functions and to prescribe a more specific training programme. The motor control problems of children with ADHD are a sadly neglected area of research and clinical attention. Working on things such as muscle strength (many with DAMP have hypotonia), body posture (often crouched and awkward), body image (usually distorted), and fine motor dysfunction (pencil grip problems, difficulties tying 
up shoe laces, eating properly) may be all important issues for the child with DAMP. Training in these areas may provide the child with a much better self-image, which, in all likelihood will benefit him/her in other areas as well.

\section{Speech-language therapy and dyslexia programmes}

Speech-language problems and dyslexia in DAMP are intimately linked. ${ }^{37}$ Any child with DAMP with an impairing speech-language disorder or with severe dyslexia will need an intervention programme specifically aimed at addressing such problems. This is not the place to go into detail in this respect, but it has to be said that the current emphasis on "ADHD" has often led to the failure to recognise language and readingwriting problems in affected children. The DAMP diagnosis makes more explicit demands on the clinician to look out for these-very common-comorbid problems in the group of disorders subsumed under the ADHD label.

\section{Psychopharmacology}

No childhood condition has better underpinned psychopharmacological treatment principles than ADHD. It is beyond doubt that central stimulants ameliorate basic symptoms of ADHD (inattention, hyperactivity, impulsivity) and also some of the associated problems (fine motor dysfunction and conduct problems). Central stimulants have been used for more than 65 years in the treatment of ADHD related problems in the USA. Millions and millions of children have received such treatment. There is no support for the hysterical claims that such well controlled treatment leads on to drug abuse or other major psychosocial problems. To the contrary, there is mounting evidence that stimulant treatment helps reduce the risk for later substance use disorder, ${ }^{38}$ and that ADHD/DAMP "in itself" if untreated is the strong predictor of substance abuse. Longer term studies indicate remaining positive effects of stimulants after one and two years of continued stimulant treatment. ${ }^{39}$ There is now also increasing scientific support for other psychopharmacological agents in the treatment of ADHD/DAMP. Thus, for instance, a new substance, atomoxetine, appears to be as positive in the treatment of ADHD as are the stimulants. ${ }^{40}$ The exact role of these substances remains unclear at the present time, but it is likely that only a few years down the road, there will be a more diverse palette when opting for psychopharmacological treatment of ADHD/DAMP.

It is my view that drugs should not be used in the treatment of DAMP unless other avenues of intervention have been entered first. In extreme cases, and particularly if change of school or dwelling has been seriously discussed, or if admission to hospital has been raised as a real issue, it would be appropriate to discuss stimulant treatment even at the "first" evaluation, after the diagnosis of DAMP has been settled. Otherwise, it would seem prudent to go for other interventions for a period of six months or so, before one opts for stimulant treatment as an addition to other interventions.

\section{Psychotherapies}

It does not appear that psychotherapy in itself is superior to non-specialised community treatment (usually equalling stimulant therapy) in ADHD. Young children (and even many adolescents) with DAMP are so unconcentrated that individual or conjoint family therapies are wasted on them. Some individuals with DAMP in late adolescence and adult life have developed so many secondary emotional problems that "psychotherapy" may be considered a necessary component of intervention. Even in such cases, it will be important for the therapist to be well versed in issues relating to ADHD and DAMP.

\section{CONCLUSIONS}

DAMP is a subgroup of ADHD—-that is, with the concomitant condition of DCD. It has been validated in several (including four population based) Scandinavian studies. The presence of DCD in ADHD predicts more academic and autistic-type problems than are generally encountered in ADHD without DCD. The severe variant of the condition affects about $1.5 \%$ of all school age children, with another few per cent afflicted by mild and moderate variants. All those with severe DAMP will need evaluation and intervention already at early school age, whereas the needs of those with more moderate DAMP are more unpredictable. The ADHD component of ADHD needs the kind of intervention generally agreed on for that condition. However, the addition of DCD and other comorbidities need to be addressed in their own right. Non-DCD comorbidities are finally beginning to receive the appropriate attention in intervention programmes for ADHD. DCD remains a "black sheep" in the history of ADHD. It is perhaps the most common (and possibly most specific) overlapping condition in ADHD; yet it is usually not even mentioned in assessment and intervention manuals for ADHD. The concept of DAMP has helped draw attention to the clinical importance of DCD in ADHD. In many ways the DAMP concept has had an-almost certainly transient-important effect in much the same way that the Asperger's syndrome concept in autism spectrum disorders helped draw attention to a very important subgroupthat with "comorbid" normal levels of IQ. Just as with Asperger's syndrome, families are often much more "at ease" with the DAMP concept than with that of ADHD, probably for the simple reason that they recognise their child and his/her many differing problems better in the diagnostic criteria for DAMP than in those for "only" ADHD. It may be that DAMP has also provided the historical link between ADHD and autism spectrum disorder, a connection which-without a doubt-will become a heated topic of debate in the next several years.

\section{REFERENCES}

1 Kadesjö B. Neuropsychiatric and neurodevelopmental disorders in a young school-age population. Epidemiology and comorbidity in a school health perspective. Göteborg: Göteborg University, 2000

2 Jensen PS. ADHD: current concepts on etiology, pathophysiology, and neurobiology. Child Adolesc Psychiatr Clin N Am 2000:9:557-72.

3 Swedish Board of Health and Welfare. ADHD hos barn och vuxna [ADHD in children and adults]. Swedish Board of Health and Welfare, 2002.

4 Clements SD. Minimal brain dysfunction in children: terminology and identification. NINDB Monograph 3. NINDB, 1966

5 Hagberg B. Minimal brain dysfunction [vad innebär det för barnets utveckling och anpassning]. Läkartidningen 1975;72:3296-300.

6 Rutter M. Psychological sequelae of brain damage in children. Am J Psychiatry 1981;138:1533-44.

7 Rutter M. Syndromes attributed to "minimal brain dysfunction" in childhood. Am J Psychiatry 1982;13:21-33.

8 Airaksinen E, Bille B, Carlström G, et al. Barn och ungdomar med $\mathrm{DAMP} / \mathrm{MBD}$ [konsensusdokument undertecknat i Göteborg den 14 september 1990]. Läkartidningen 1991;9:714.

9 Gillberg C. Nordisk enighet om DAMP/ADHD [Aktuellt dokument sammanfattar dagens kunskap]. Läkartidningen 1999;28:3330-1.

10 Gillberg C, Rasmussen P, Carlström G, et al. Perceptual, motor and attentional deficits in six-year-old children. Epidemiological aspects. J Child Psychol Psychiatry 1982;23:131-44.

11 Kadesjö B, Gillberg C. Attention deficits and clumsiness in Swedish 7-year-old children. Dev Med Child Neurol 1998;40:796-811.

12 Landgren $M$, Pettersson R, Kjellman B, et al. ADHD, DAMP and other neurodevelopmental/neuropsychiatric disorders in six-year-old children. Epidemiology and comorbidity. Dev Med Child Neurol 1996;38:891-906.

13 Kadesiö B, Gillberg C. Developmental coordination disorder in Swedish 7-year-old children. J Am Acad Child Adolesc Psychiatry 1999:38:820-8.

14 Kopp S, Gillberg C. Swedish child and adolescent psychiatric out-patients: a five year cohort. Eur Child Adolesc Psychiatry $2003 ; 12: 30-5$

15 Kadesjö B, Gillberg C. The comorbidity of ADHD in the general population of Swedish school-age children. J Child Psychol Psychiatry 2001:42:487-92.

16 Gillberg C. Perceptual, motor and attentional deficits in Swedish primary school children. Some child psychiatric aspects. J Child Psychol Psychiatry 1983;24:377-403.

17 Gillberg IC, Gillberg C. Asperger syndrome-some epidemiological considerations: a research note. J Child Psychol Psychiatry 1989;30:631-8. 
18 Rasmussen P Gillberg C. Natural outcome of ADHD with developmental coordination disorder at age 22 years: a controlled, longitudinal, community-based study. J Am Acad Child Adolesc Psychiatry 2000;39:1424-31.

19 Soorani-Lunsing RJ, Hadders-Algra M, Touwen BC. Do girls with minor neurological dysfunction mature at a later age? Acta Paediatr 1998:87:117-18

20 Hadders-Algra $M$. Two distinct forms of minor neurological dysfunction: perspectives emerging from a review of data of the Groningen Perinatal Project. Dev Med Child Neurol 2002;44:561-71.

21 Gillberg C, Rasmussen P. Perceptual, motor and attentional deficits in seven-year-old children: background factors. Dev Med Child Neurol 1982;24:752-70.

22 Landgren $M$, Kjellman B, Gillberg C. Attention deficit disorder with developmental coordination disorders. Arch Dis Child 1998;79:207-12.

23 Whitaker AH, Van Rossem R, Feldman JF, et al. Psychiatric outcomes in low-birth-weight children at age 6 years: relation to neonatal cranial ultrasound abnormalities. Arch Gen Psychiatry 1997;54:847-56.

24 Aronson M, Hagberg B, Gillberg C. Attention deficits and autistic spectrum problems in children exposed to alcohol during gestation: a follow-up study. Dev Med Child Neurol 1997;39:583-7.

25 Mick E, Biederman J, Faraone SV, et al. Case-control study of attention-deficit hyperactivity disorder and maternal smoking, alcohol use, and drug use during pregnancy. J Am Acad Child Adolesc Psychiatry 2002;41:378-85.

26 Swanson JM, Flodman P, Kennedy J, et al. Dopamine genes and ADHD. Neurosci Biobehav Rev 2000;24:21-5.

27 Jensen $\mathbf{P}$. Longer term effects of stimulant treatments for attention-deficit/hyperactivity disorder. Journal of Attention Disorders 2002;6(suppl 1):S45-56

28 Smalley SL, Kustanovich V, Minassian SL, et al. Genetic linkage of attention-deficit/hyperactivity disorder on chromosome 16p13, in a region implicated in autism. Am J Hum Genet 2002;7:959-63.

29 Pereira HS, Landgren M, Gillberg C, et al. Parametric control of finger-tip forces during precision grip lifts in children with DCD (developmental coordination disorder) and DAMP (deficits in attention motor control and perception). Neuropsychologia 2001;39:478-88.

30 Nydén A, Billstedt E, Hjelmquist E, et al. Neurocognitive stability in Asperger syndrome, ADHD reading and writing disorder: a pilot study. Dev Med Child Neurol 2001:43:165-71.

31 DuPaul GJ, Power TJ, Anastopoulos AD, et al. ADHD Rating Scale-IV Checklists, norms, and clinical interpretation. New York: Guilford Press, 1998.

32 Achenbach TM. Manual for the Child Behavior Checklist/4-18 and 1991 profile. University of Vermont, 1991

33 Gillberg C, Carlström G, Rasmussen P, et al. Perceptual, motor and attentional deficits in seven-year-old children. Neurological screening aspects. Acta Paediatr Scand 1983;72:119-24.

34 Waldrop MF, Halverson CFJ. Minor physical anomalies and hyperactive behaviour in young children. In: Helmuth J, ed. The exceptional infant. New York: Brunner-Mazel, 1971:343-80.

35 Barkley RA. Attention deficit hyperactivity disorder: a handbook for diagnosis and treatment, 2nd edn. New York: Guilford Press, 1998.

36 Adler H. Children with problems in physical education in middle school need help and assistance after grade school [in Swedish]. Läkartidningen 1982:79:2878-80

37 Rasmussen P, Gillberg C, Waldenström E, et al. Perceptual, motor and attentional deficits in seven-year-old children: neurological and neurodevelopmental aspects. Dev Med Child Neurol 1983;25:315-33.

38 Biederman J, Wilens TE, Mick E, et al. Does attention-deficit hyperactivity disorder impact the developmental course of drug and alcohol abuse and dependence? Biol Psychiatry 1998;44:269-73.

39 Gillberg C, Melander H, von Knorring A-L, et al. Long-term stimulant treatment of children with attention-deficit hyperactivity disorder symptoms. A randomized, double-blind, placebo-controlled trial. Arch Gen Psychiatry 1997;54:857-64.

40 Buitelaar J, Dankaerts M, Gillberg C, et al. A prospective, multicenter open-label assessment of atomoxetine in non-North American children and adolescents with ADHD. Eur Child Adolesc Psychiatry. In press.

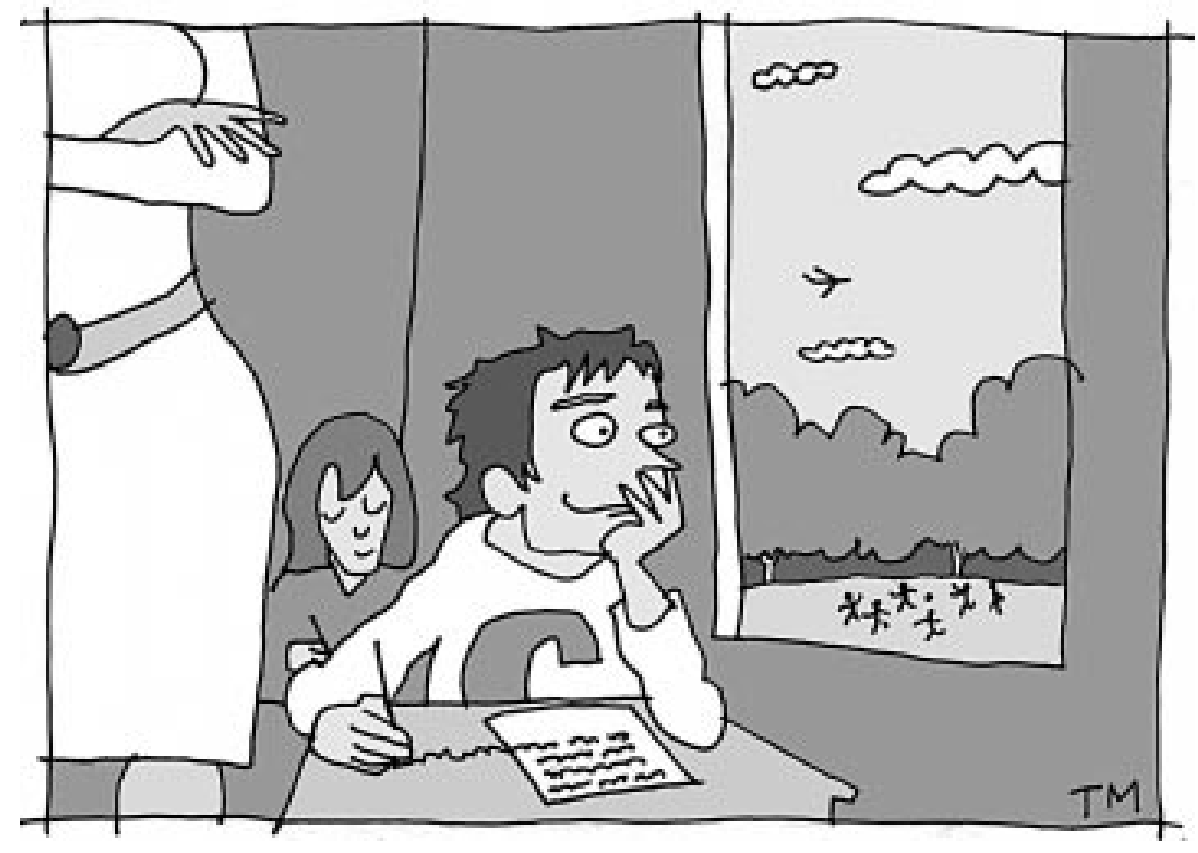

Illustration by Terry McElroy. 